\title{
Perspectives in in situ transmission electron microscopy studies on lithium battery electrodes
}

\author{
Hyun-Wook Lee ${ }^{1,2}$, Yuzhang $\mathrm{Li}^{1}$, Yi Cui ${ }^{1,3^{*}}$
}

${ }^{1}$ Department of Materials Science and Engineering, Stanford University, Stanford, California 94305, United States

2 School of Energy and Chemical Engineering, Ulsan National Institute of Science and Technology (UNIST), Ulsan 44919, Republic of Korea

${ }^{3}$ Stanford Institute for Materials and Energy Sciences, SLAC National Accelerator Laboratory, 2575 Sand Hill Road, Menlo Park, California 94025, United States

*Corresponding author. E-mail: yicui@stanford.edu 


\begin{abstract}
In situ transmission electron microscopy (TEM) has emerged as a promising characterization tool for the observation of nanoscale processes in lithium ion batteries. TEM offers direct visualization of lithiation during electrochemical reaction combined with atomic spatial and temporal resolution imaging, selected area diffraction (SAD), electron energy-loss spectroscopy (EELS), and energy dispersive X-ray spectroscopy (EDS). This review highlights recent progress on in situ TEM studies on lithium ion battery electrodes associated with open and closed cell configurations. The advantages and challenges of each cell configuration are addressed. These techniques allow for new discoveries and a better fundamental understanding of energy related technologies.
\end{abstract}

\title{
Introduction
}

The high demands for rechargeable lithium ion batteries (LIBs) have significantly increased each year for mobile electronics, electric vehicles, and stationary energy storage over the past two decades as a convenient power source [1-3]. In order to fulfill the demands in these applications, tremendous research progress has been made at stabilizing new electrode materials with high performance and identifying the fundamental failure mechanisms that prevent a prolonged cycle life. Alloy and conversion materials with high specific capacity like silicon, sulfur, and conversion metal oxides have the most promise to increase the energy density of LIBs, but also have scientific challenges to retain their theoretical capacities upon repeated charge and discharge cycles [4-7]. These new electrode materials have created a high interest for experimental techniques that are able to provide direct, atomic-scale, and real-time observation during the electrochemical processes. Such demands lead to extensive researches about in situ X- 
ray diffractography [8,9], Raman [10,11], Fourier transform infrared spectroscopy (FTIR) [12], and nuclear magnetic resonance (NMR) [13,14], etc.

Among the direct characterization tools, an in situ transmission electron microscopy (TEM) offers exceptional opportunities for monitoring the dynamic processes of various electrode materials during electrochemical reaction at high spatial and temporal resolution [1517]. Depending on the experimental set up (open or closed cell configuration), the structural change of electrodes or the interface between a liquid electrolyte and a solid electrode can be observed upon battery charge/discharge cycles. This can bring new observations of reaction processes to light and provide a deep understanding for the fundamentals of a new electrode material's battery chemistry. The development of the in situ TEM characterization also benefits from the technology of TEM instrument combined with aberration corrected high-resolution imaging, electron energy-loss spectroscopy (EELS), and energy dispersive X-ray spectroscopy (EDS) which have enabled many discoveries in dynamic materials processes at the atomic level with reliable chemical information.

\section{Open-cell configuration using a bias holder}

In a typical battery system, a battery consists of a positive and negative electrode and an organic liquid electrolyte. To mimic the system inside TEM, the demonstration of a liquid electrolyte was initially a formidable challenge because the TEM column needs to maintain a high vacuum level of about $10^{-5} \mathrm{~Pa}$. Since typical carbonate type electrolytes cannot be directly used due to a high vapor pressure, ionic liquid electrolytes with very low vapor pressures were used to enable a successful demonstration without compromising the TEM instrument [16]. Another open-cell configuration is conducted by a solid electrolyte [18]. A naturally formed 
lithium oxide layer is extensively used for the nanobattery for in situ TEM characterization, which is generated by a short exposure to air when lithium metal is transferred from an inert gas glovebox into the TEM. The common features of these two approaches are that they are both experimentally simple, less limited on the sample size, and especially capable to acquire atomicscale images and video.

Figure 1(a) illustrates the schemes of the open-cell configuration for both solid and liquid electrolyte configuration [18]. A two-electrode system is used for in situ TEM observation, including the sample-loaded working electrode and the counter electrode for lithium source. As shown in Figure 1(a), both ionic liquid and solid electrolytes generally contact the tip of the sample and the electrochemical reaction occurs at the interface of the materials, which enables the electron beam to pass through the sample to observe samples with atomic-scale resolution. Many new phenomena have been directly visualized with the clearest evidence and reaction mechanisms have been proposed. Furthermore, the structural and chemical analyses to verify elemental distribution or binding energy are also more suitable using selected area diffraction (SAD), EDS, and EELS during or after the reaction due to an unblocked position of the sample $[19,20]$.

For instance, Si nanostructures have been intensively studied as the most promising anode material for Li ion battery due to a high energy density with relatively low reaction potential. However, battery decay due to volume expansion and fracture during the lithiation process have led many researchers to engineer nanostructures and improve the fundamental understanding of reaction mechanism with electrochemical and mechanical characterization in the hopes of stabilizing silicon. As shown in Figure 1(b) and (c), the open-cell configuration revealed the preferential lithiation direction of silicon ( $\mathrm{Si}$ ) nanowires along the $<110>$ crystal 
orientation with favored fracture sites between them, and also showed atomic resolution video upon electrochemical reaction [21]. Furthermore, this configuration illustrated the particle shape of crystalline and as well as amorphous Si with dynamic and clear evidence [22-24]. In addition, this in situ TEM characterization also enables particle-interaction studies on Si nanopillars, which has drastically different behavior as compared to a single particle reaction of $\mathrm{Si}$ nanostructure in Figure 1(d) and (e) [25]. Once the preferential lithiation expansion in the $<110>$ orientations is blocked, the second preferential lithiation expansion in the $<100>$ crystal orientation occurred which also altered the fracture orientation and sizes. Consequently, the open-cell configuration enables the investigation of dynamic lithiation and delithation processes of electrode materials with more apparent motion and chemical characterizations.

\section{An electrochemical liquid-cell configuration using silicon/silicon nitride membrane chips}

Although the open-cell configuration has provided plenty of electrochemical process observations on the electrode materials, there are several shortcomings: (1) the ionic liquid and solid electrolytes are not common electrolytes for Li ion batteries such as organic carbonates; (2) the contact between an electrode and an solid electrolyte occurs only partially which gives inhomogeous reaction on the electrode; (3) the relatively large overpotential is accompanied to overcome the resistance of the solid electrolyte that the different electrochemical reaction may be observed; and (4) the investigation of interface is unavailable to understand the reaction between an electrode and the contacted electrolyte. To improve these drawbacks, electrolyte-enclosed configurations have been developed using $\mathrm{Si}$ membrane chips for observation of an electrochemical reaction on electrodes enabling under TEM and so-called "liquid-cell" [17,2628]. The first demonstration of an electrochemical liquid cell was invented by F. M. Ross et al., 
which observed the deposition of electrochemically generated $\mathrm{Cu}$ nanoparticles using a $\mathrm{Si}$ membrane chip inside TEM [15]. Through the initial breakthrough and the knowledge from liquid cells which were observed nanoparticles in solution inside a TEM column, different electrochemical liquid cells have been developed to enable the more genuine electrochemical processes with easier assembly, more reproducibility, and more durability under TEM.

A typical electrochemical liquid-cell configuration consists of an electrochemical microchip and a spacer microchip fabricated by a Si wafer as shown in Figure 2(a) and (b). $\mathrm{SiN}_{x}$ membrane deposited onto a $\mathrm{Si}$ wafer becomes an electron beam channel to observe the samples and the Si wafer supports this membrane window. Initially, the electrochemical lithiation and delithiation processes of $\mathrm{Cu}$-coated $\mathrm{Si}$ nanowire were performed using the closed liquid-cell. Figure 2(c) shows the structural evolution of the nanowire. Compared to the result using opencell configuration in Figure 1(b), the Si nanowire in Figure 2(c) shows the homogeneous expansion along the side wall of the nanowire resulting from the more Li paths by immersion to the electrolyte. Moreover, since the liquid cell is more suitable to observe the interface reaction, initial several works $[27,29,30]$ focused on performing Li-metal dendritic and solid electrolyte interface (SEI) layer formations as shown in Figure 2(d) as an example. Although the formation of SEI layers are not clearly verified yet due to the controversy of electrolyte deposition onto the electrode, the attempts shed new light on the possible causes.

The liquid cell has also explored the lithiation and delithiation behaviors of electrodes by use of the techniques of energy filtered TEM (EFTEM)-based spectroscopic imaging [26], EELS [31], and EDS [32]. Muller et al. [26] investigated degradation mechanisms of $\mathrm{LiFePO}_{4}$ particles using EFTEM images during electrochemical charge and discharge cycles in an aqueous electrolyte. By selecting a valence energy loss peak at $5 \mathrm{eV}$ that offers the information of $\mathrm{FePO}_{4}$, 
lithiation state of a $\mathrm{LiFePO}_{4}$ electrode and surrounding aqueous electrolyte was determined. Another approach to look at the lithation state of electrodes which has an insertion mechanism was done by Unocic et al. [31]. They provided EELS technique to thin film battery electrodes of $\mathrm{LiMn}_{2} \mathrm{O}_{4}$ and $\mathrm{Li}_{4} \mathrm{Ti}_{5} \mathrm{O}_{12}$ and observed the change of oxidation state of transition metals during electrochemical reaction. Although the liquid cell contained an organic electrolyte, tracking of electrochemical redox processes was achievable. Also, Zheng et al. [32] studied a conversion electrode of $\mathrm{MoS}_{2}$ using conventional $\mathrm{LiPF}_{6} / \mathrm{EC} / \mathrm{DEC}$ electrolyte. They illustrated the conversion reaction of $\mathrm{MoS}_{2}$ near $1.1 \mathrm{~V}$ that also confirmed by nanobeam diffraction. During the reaction, $\mathrm{Ti}$ anode created SEI layer characterized by EDS and nanobeam diffraction in order to verify the composition. Therefore, despite of the electrolyte decomposition mentioned above, these chemical analyses to a liquid cell support the electrochemical reaction associated with the limitation by images.

\section{Challenges and opportunities of in situ TEM studies}

In situ TEM on battery electrodes is becoming a feasible characterization tool to understand the behavior inside electrodes using very direct structural and chemical evolution. With ongoing development of TEM technology and in situ holders, the future in situ TEM studies can be much more similar to real reaction conditions but still need to improve the challenges below. First, ionization effect of the electron beam should be considered especially to closed cell configuration. The organic electrolyte is sensitive to electron beam, typically for the acceleration voltage of 60 to $300 \mathrm{kV}$, resulting in decomposition of the electrolyte as shown in Figure 3(a) [33]. As a result, the $\operatorname{SiN}_{x}$ membrane windows in a closed liquid cell are bulging out. According to Muller et al. [34] measurement in Figure 3(b), the center of window thickness 
expands about 2 times higher than the initial thickness when aqueous medium is inside. Even Li ion in an electrode material could be damaged by highly intense beam at high resolution magnification. So, the effect of the dose rate to the nanobattery should be considered and optimized. Second, the kinetics of both open and closed cell configurations inside TEM are more sensitive according to the electrolyte condition than the electrodes. Open cell configuration conducted by $\mathrm{Li}_{2} \mathrm{O}$ solid electrolyte needs overpotential to overcome the solid electrolyte. Also, the current set-up in closed cell configuration needs relatively long distance between the two electrode materials due to the parallel geometry of the samples. Thus, the less effect of the electrolytes will be a difficult issue to mimic a real battery.

These many challenges also offer the opportunities to develop in situ TEM technology. For example, the fast capturing cameras, for example One-view and $\mathrm{K} 2$, have a higher chance to acquire the precise reaction with less electron beam effect in Figure 3(c) [35]. Knowledge and development from the observation on nanoparticles in liquid phase [36-39] can become examples to overcome challenges of in situ TEM technologies on Li ion batteries. An enhanced cell configuration can also lessen the damages. For example, Andrew J. Leenheer et al. demonstrated the manipulated liquid cell as shown in Figure 3(d) [40]. In addition, with the intrinsic functions of SAD, EELS, EDS, and more characterization tools in TEM, in situ TEM characterization will continue to provide new understandings of battery chemistry to resolve the issues and suggest materials to advance battery performance to be utilized in our daily life.

\section{References and recommend reading}

Papers of particular interest, published within the period of review, have been highlighted as: normal, special interest $(*)$, outstanding interest $(* *)$ 
[1] M. Armand, J.-M. Tarascon, Building better batteries, Nature. 451 (2008) 652-657. http://www.ncbi.nlm.nih.gov/pubmed/18256660.

[2] B. Dunn, H. Kamath, J.-M. Tarascon, Electrical energy storage for the grid: a battery of choices., Science. 334 (2011) 928-935. doi:10.1126/science.1212741.

[3] P.G. Bruce, S.A.S. Freunberger, L.J. Hardwick, J.-M. Tarascon, Li-O2 and Li-S batteries with high energy storage, Nat. Mater. 11 (2012) 19-29. doi:10.1038/NMAT3191.

[4] C.K. Chan, H. Peng, G. Liu, K. Mcllwrath, X.F. Zhang, R.A. Huggins, et al., Highperformance lithium battery anodes using silicon nanowires, Nat. Nanotechnol. 3 (2008) 31-35. http://www.nature.com/nnano/journal/v3/n1/full/nnano.2007.411.html.

[5] N. Liu, Z. Lu, J. Zhao, M.T. McDowell, H.-W. Lee, W. Zhao, et al., A pomegranateinspired nanoscale design for large-volume-change lithium battery anodes, Nat. Nanotechnol. 9 (2014) 187-192. doi:10.1038/nnano.2014.6.

[6] X. Ji, K.T. Lee, L.F. Nazar, A highly ordered nanostructured carbon-sulphur cathode for lithium-sulphur batteries., Nat. Mater. 8 (2009) 500-506. doi:10.1038/nmat2460.

[7] P. Poizot, S. Laruelle, S. Grugeon, L. Dupont, J.-M. Tarascon, Nano-sized transition-metal oxides as negative-electrode materials for lithium-ion batteries, Nature. 407 (2000) 496499. doi: $10.1038 / 35035045$.

[8] J.R. Dahn, R.R. Haering, Anomalous bragg peak widths in LixTiS2, Solid State Commun. 40 (1981) 245-248. doi:10.1016/0038-1098(81)90750-X.

[9] A.S. Andersson, B. Kalska, L. Haggstrom, J.O. Thomas, Lithium extraction/insertion in LiFePO4: An X-ray diffraction and Mössbauer spectroscopy study, Solid State Ionics. 130 (2000) 41-52. doi:10.1016/S0167-2738(00)00311-8. 
[10] T. Itoh, H. Sato, T. Nishina, T. Matue, I. Uchida, In situ Raman spectroscopic study of LixCoO2 electrodes in propylene carbonate solvent systems, J. Power Sources. 68 (1997) 333-337. doi:10.1016/S0378-7753(97)02539-1.

[11] K. Dokko, M. Mohamedi, N. Anzue, T. Itoh, I. Uchida, In situ Raman spectroscopic studies of LiNixMn2-xO4 thin film cathode materials for lithium ion secondary batteries, J. Mater. Chem. 12 (2002) 3688-3693. doi:10.1039/b206764a.

[12] E. Goren, O. Chusid, D. Aurbach, The Application of In Situ FTIR Spectroscopy to the Study of Surface Flims Formed on Lithium and Noble Metals at Low Potentials in Li Battery Electrolytes, J. Electrochem. Soc. 138 (1991) L6-L9.

[13] M. Letellier, F. Chevallier, F. Béguin, E. Frackowiak, J.-N. Rouzaud, The first in situ 7Li NMR study of the reversible lithium insertion mechanism in disorganised carbons, J. Phys. Chem. Solids. 65 (2004) 245-251. doi:10.1016/j.jpcs.2003.10.022.

[14] B. Key, R. Bhattacharyya, M. Morcrette, V. Seznéc, J.-M. Tarascon, C.P. Grey, et al., Real-time NMR investigations of structural changes in silicon electrodes for lithium-ion batteries., J. Am. Chem. Soc. 131 (2009) 9239-49. doi:10.1021/ja8086278.

[15] M.J. Williamson, R.M. Tromp, P.M. Vereecken, R. Hull, F.M. Ross, Dynamic microscopy of nanoscale cluster growth at the solid-liquid interface, Nat. Mater. 2 (2003) 532-536. doi:10.1038/nmat944.

** This paper describes the first demonstration of a liquid cell with an electrochemical reaction inside TEM.

[16] J.Y. Huang, L. Zhong, C.M. Wang, J.P. Sullivan, X. Wu, L.Q. Zhang, et al., In Situ Observation of the Electrochemical Lithiation of a Single SnO2 Nanowire Electrode, Science. 336 (2010) 1515-1520. doi:10.1126/science.1195628. 
** This paper describes the first observation of the electrochemical lithiation using open cell configuration in TEM.

[17] M. Gu, L.R. Parent, B.L. Mehdi, R.R. Unocic, M.T. McDowell, R.L. Sacci, et al., Demonstration of an electrochemical liquid cell for operando transmission electron microscopy observation of the lithiation/delithiation behavior of Si nanowire battery anodes, Nano Lett. 13 (2013) 6106-6112. doi:10.1021/nl403402q.

** This paper describes the first demonstration to observe an anode material in Li ion battery.

[18] X.H. Liu, H. Zheng, L. Zhong, S. Huang, K. Karki, L.Q. Zhang, et al., Anisotropic swelling and fracture of silicon nanowires during lithiation, Nano Lett. 11 (2011) 33123318. doi:10.1021/nl201684d.

[19] X.H. Liu, L.Q. Zhang, L. Zhong, Y. Liu, H. Zheng, J.W. Wang, et al., Ultrafast electrochemical lithiation of individual Si nanowire anodes, Nano Lett. 11 (2011) 22512258. doi:10.1021/nl200412p.

[20] C.-M. Wang, X. Li, Z. Wang, W. Xu, J. Liu, F. Gao, et al., In situ TEM investigation of congruent phase transition and structural evolution of nanostructured silicon/carbon anode for lithium ion batteries., Nano Lett. 12 (2012) 1624-32. doi:10.1021/n1204559u.

[21] X.H. Liu, J.W. Wang, S. Huang, F. Fan, X. Huang, Y. Liu, et al., In situ atomic-scale imaging of electrochemical lithiation in silicon., Nat. Nanotechnol. 7 (2012) 749-756. doi:10.1038/nnano.2012.170.

[22] X.H. Liu, L. Zhong, S. Huang, S.X. Mao, T. Zhu, J.Y. Huang, Size-dependent fracture of silicon nanoparticles during lithiation, ACS Nano. 6 (2012) 1522-1531.

[23] M.T. McDowell, I. Ryu, S.W. Lee, C. Wang, W.D. Nix, Y. Cui, Studying the kinetics of crystalline silicon nanoparticle lithiation with in situ transmission electron microscopy, 
Adv. Mater. 24 (2012) 6034-6041. doi:10.1002/adma.201202744.

** This paper describes the first observation of the micron sized Si fracture upon eletrochemical lithiation inside TEM.

[24] M.T. McDowell, S.W. Lee, J.T. Harris, B. a Korgel, C. Wang, W.D. Nix, et al., In situ TEM of two-phase lithiation of amorphous silicon nanospheres, Nano Lett. 13 (2013) 758-764. doi:10.1021/nl3044508.

* This paper describes the first observation of amorphous Si lithiation upon eletrochemical reaction inside TEM.

[25] S.W. Lee, H.-W. Lee, I. Ryu, W.D. Nix, H. Gao, Y. Cui, Kinetics and fracture resistance of lithiated silicon nanostructure pairs controlled by their mechanical interaction, Nat. Commun. 6 (2015) 7533. doi:10.1038/ncomms8533.

[26] M.E. Holtz, Y. Yu, D. Gunceler, J. Gao, R. Sundararaman, K. a. Schwarz, et al., Nanoscale imaging of lithium ion distribution during in situ operation of battery electrode and electrolyte, Nano Lett. 14 (2014) 1453-1459. doi:10.1021/n1404577c.

** This paper describes the first observation of lithiation and delithiation of $\mathrm{LiFePO}_{4}$ using EFTEM technique in a liquid cell.

[27] Z. Zeng, W.-I. Liang, H. Liao, H.L. Xin, Y.-H. Chu, H. Zheng, Visualization of Electrode-Electrolyte Interfaces in LiPF6/EC/DEC Electrolyte for Lithium Ion Batteries via in Situ TEM, Nano Lett. 14 (2014) 1745-1750. doi:10.1021/n1403922u.

** This paper describes the first demonstration of lithium metal dendrite and SEI formation in a commercial eletroylte.

[28] R.R. Unocic, R.L. Sacci, G.M. Brown, G.M. Veith, N.J. Dudney, K.L. More, et al., Quantitative Electrochemical Measurements Using In Situ ec-S/TEM Devices, Microsc. 
Microanal. 20 (2014) 452-461. doi:10.1017/S1431927614000166.

[29] R.L. Sacci, J.M. Black, N. Balke, N.J. Dudney, K.L. More, R.R. Unocic, Nanoscale imaging of fundamental li battery chemistry: solid-electrolyte interphase formation and preferential growth of lithium metal nanoclusters., Nano Lett. 15 (2015) 2011-8. doi:10.1021/n15048626.

* This paper describes the observation of lithium metal dendrite and SEI formation supported by STEM in a commercial eletroylte.

[30] B.L. Mehdi, J. Qian, E. Nasybulin, C. Park, D. a. Welch, R. Faller, et al., Observation and Quantification of Nanoscale Processes in Lithium Batteries by Operando Electrochemical (S)TEM, Nano Lett. 15 (2015) 2168-2173. doi:10.1021/acs.nanolett.5b00175.

[31] R.R. Unocic, L. Baggetto, G.M. Veith, J.A. Aguiar, K.A. Unocic, R.L. Sacci, et al., Probing battery chemistry with liquid cell electron energy loss spectroscopy., Chem. Commun. 51 (2015) 16377-16380. doi:10.1039/c5cc07180a.

[32] Z. Zeng, X. Zhang, K. Bustillo, K. Niu, C. Gammer, J. Xu, et al., In Situ Study of Lithiation and Delithiation of $\mathrm{MoS}_{2}$ Nanosheets Using Electrochemical Liquid Cell Transmission Electron Microscopy, Nano Lett. 15 (2015) 5214-5220. doi:10.1021/acs.nanolett.5b02483.

[33] P. Abellan, B.L. Mehdi, L.R. Parent, M. Gu, C. Park, W. Xu, et al., Probing the degradation mechanisms in electrolyte solutions for li-ion batteries by in situ transmission electron microscopy, Nano Lett. 14 (2014) 1293-1299. doi:10.1021/n1404271k.

[34] M.E. Holtz, Y. Yu, J. Gao, H.D. Abruna, D. a. Muller, In Situ Electron Energy Loss Spectroscopy in Liquids, Microsc. Microanal. 19 (2013) 1027-1035. http://journals.cambridge.org/production/action/cjoGetFulltext?fulltextid=8930592. 
[35] T. LaGrange, B.W. Reed, D.J. Masiel, Movie-mode dynamic electron microscopy, MRS Bull. 40 (2015) 22-28. doi:10.1557/mrs.2014.282.

[36] H.M. Zheng, R.K. Smith, Y.W. Jun, C. Kisielowski, U. Dahmen, A.P. Alivisatos, Observation of Single Colloidal Platinum Nanocrystal Growth Trajectories, Science. 324 (2009) 1309-1312. doi:10.1126/science.1172104.

[37] J.M. Yuk, J. Park, P. Ercius, K. Kim, D.J. Hellebusch, M.F. Crommie, et al., HighResolution EM of Colloidal Nanocrystal Growth Using Graphene Liquid Cells, Science. 336 (2012) 61-64.

[38] H.-G. Liao, D. Zherebetskyy, H. Xin, C. Czarnik, P. Ercius, H. Elmlund, et al., Facet development during platinum nanocube growth, Science. 345 (2014) 916-919. doi:10.1126/science.1253149.

[39] J. Park, H. Elmlund, P. Ercius, J.M. Yuk, D.T. Limmer, Q. Chen, et al., 3D structure of individual nanocrystals in solution by electron microscopy, Science. 349 (2015) 290-295.

[40] A.J. Leenheer, J.P. Sullivan, M.J. Shaw, C.T. Harris, A Sealed Liquid Cell for In Situ Transmission Electron Microscopy of Controlled Electrochemical Processes, Microelectromechanical Syst. J. PP (2015) 1. doi:10.1109/JMEMS.2014.2380771.

\section{Acknowledgements}

Y.C. acknowledges the support from the Assistant Secretary for Energy Efficiency and Renewable Energy, Office of Vehicle Technologies of the US Department of Energy. H.-W.L. acknowledges support from the Basic Science Research Program through the National Research Foundation of Korea (NRF) funded by the Ministry of Education, Science and Technology under NRF-2012R1A6A3A03038593. 


\section{Figure legends}

Figure 1. In situ TEM studies using open-cell configuration. (a) Schematic diagram of in situ experimental setup on $\mathrm{Si}$ Nanowire sample using solid electrolyte of $\mathrm{Li}_{2} \mathrm{O}$ and ionic liquid electrolyte. (b) Morphology evolution of the Si nanowire during lithiation in the solid cell. ((a) and (b): Reprinted with permission from Ref. 18. Copyright 2011 American Chemical Society.) (c) High-resolution TEM image showing the lithiation process by lateral ledge flow. (Reprinted by permission from Macmillan Publishers Ltd: Ref. 21, copyright 2012) (d) SEM and TEM images of Si pillar interaction research. All scale bars in SEM and TEM images are $500 \mathrm{~nm}$. (e) The diameters of crystalline $\mathrm{Si}$ core and lithiated outer $\mathrm{Li}_{x} \mathrm{Si}$ for the time line in the middle of lithiation. The lithiation cannot proceed further along $<110>$ direction against the neighboring pillars due to the mechanical clamping. ((d) and (e): Reprinted by permission from Macmillan Publishers Ltd: Ref. 25, copyright 2015)

Figure 2. In situ TEM studies using closed-cell configuration. Schematic diagram of in situ liquid electrochemical setup depicting (a) the assembly of the Si membrane chip devices within the tip of the in situ TEM holder and (Reprinted by permission from Cambridge University Press: Ref. 28, copyright 2014) (b) Si Nanowire sample inside Si membrane chips using organic liquid electrolyte. (c) Morphology evolution of the Si nanowire during lithiation in the close-cell. ((b) and (c): Reprinted with permission from Ref. 17. Copyright 2013 American Chemical Society.) (d) Another schematic diagram of in situ liquid cell configuration and TEM images of the growth of solid electrolyte interface film and lithium metal dendrite growth. (Reprinted with permission from Ref. 27. Copyright 2014 American Chemical Society.) 
Figure 3. Challenges and opportunities in in situ TEM. (a) Schematic diagram of electron beam damage on the organic liquid electrolyte. (Reprinted with permission from Ref. 33. Copyright 2014 American Chemical Society.) (b) Liquid thickness due to the bulging of the close-cell in the fluid stage. (Reprinted by permission from Cambridge University Press: Ref. 34, copyright 2013) (c) Schematic of movie-mode dynamic TEM. (Reprinted by permission from Cambridge University Press: Ref. 35, copyright 2015) (d) Schematic illustration and its photograph of another type of a liquid cell containing alignment beads. (Reprinted by permission from IEEE: Ref. 40, copyright 2015) 
Figure 1

Open-cell configuration

(a)

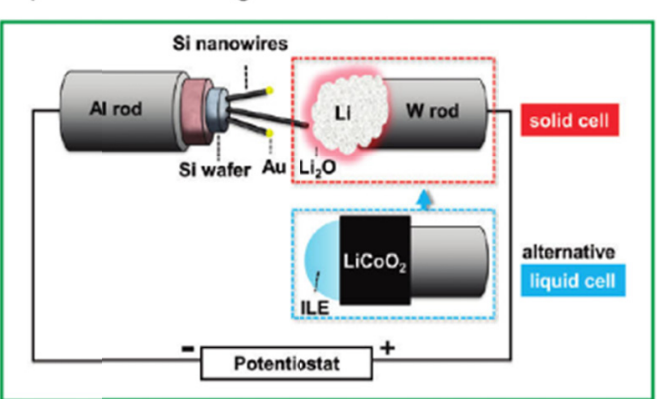

(c)

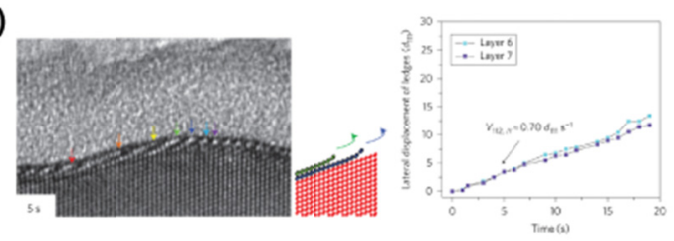

(d)

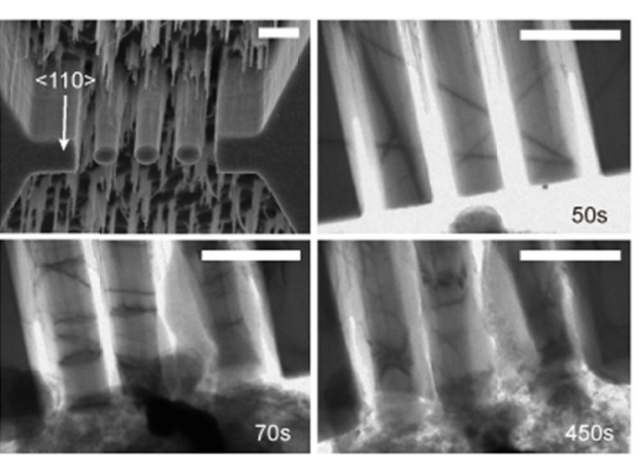

(b)

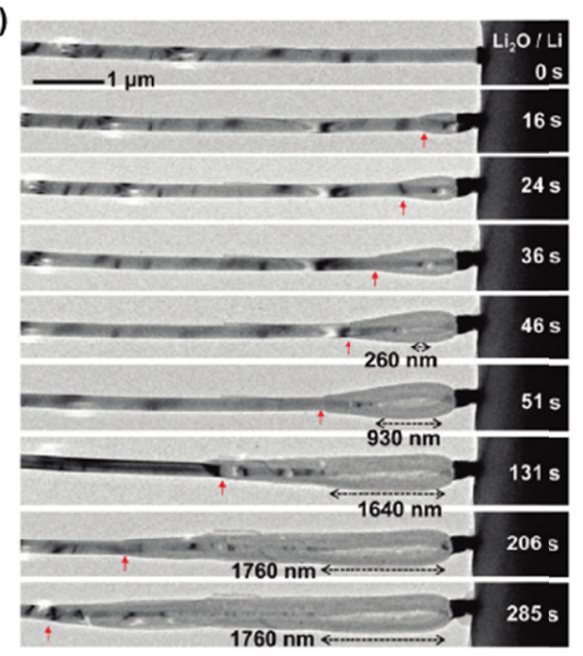

(e)

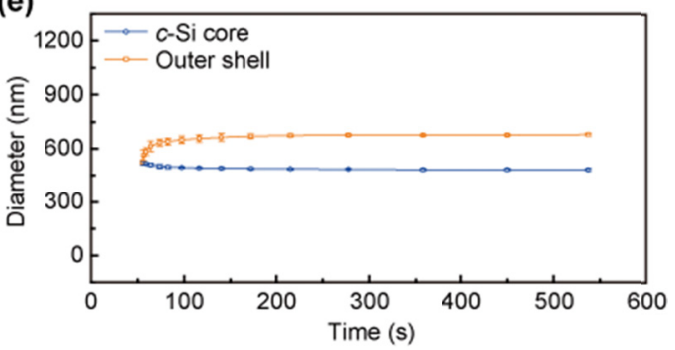


Figure 2

Liquid-cell configuration

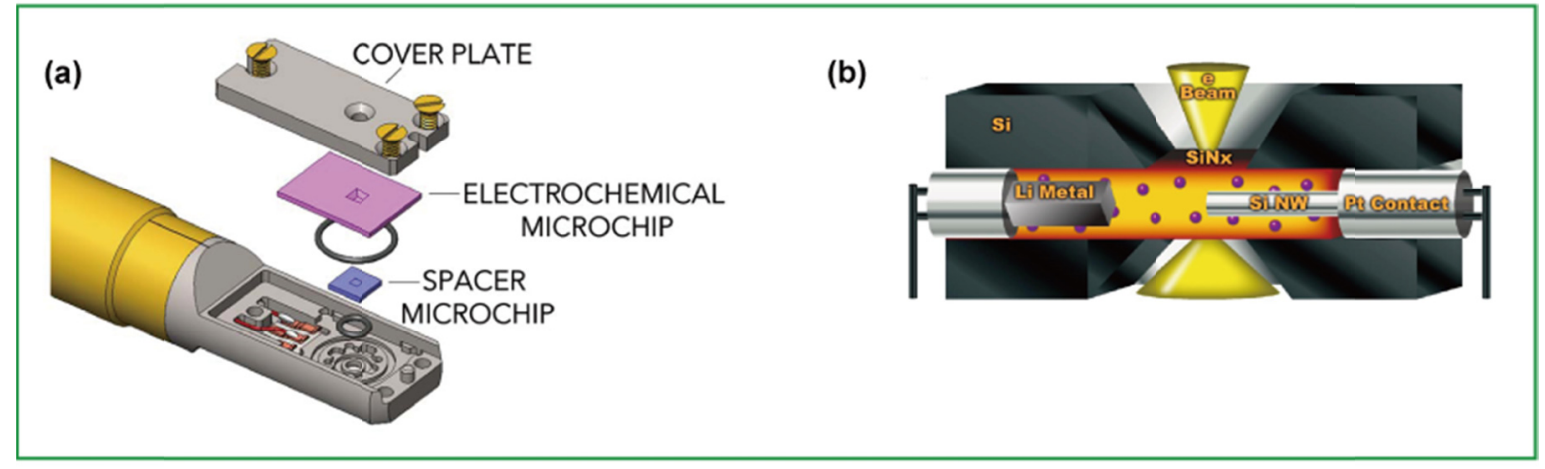

(c)

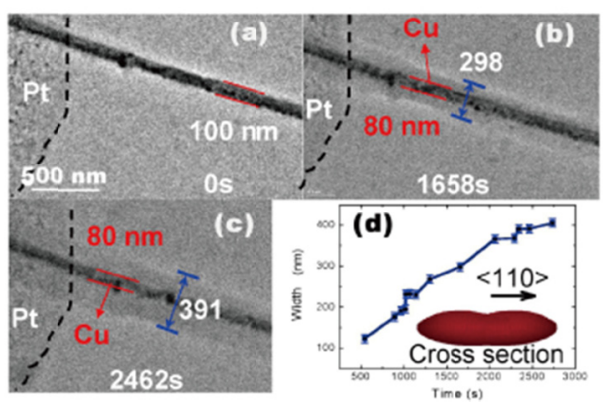

(d)

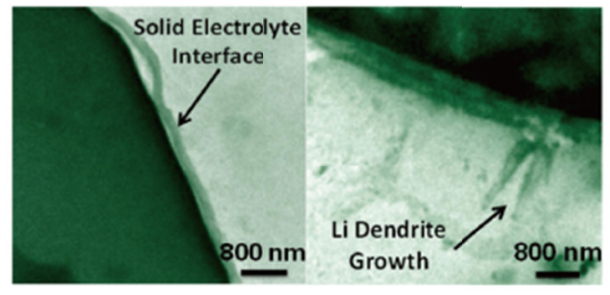


Figure 3

(a)

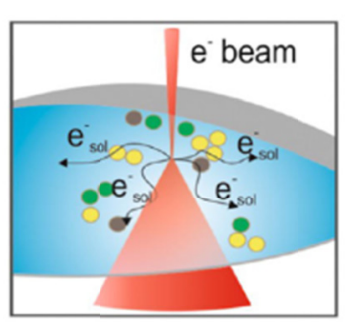

(b)

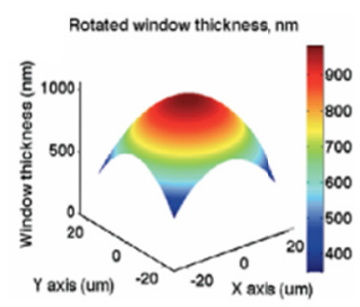

(c)

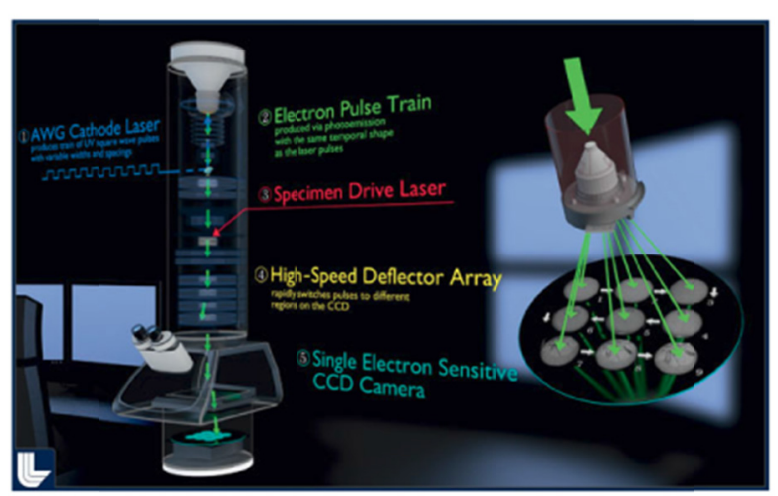

(d)

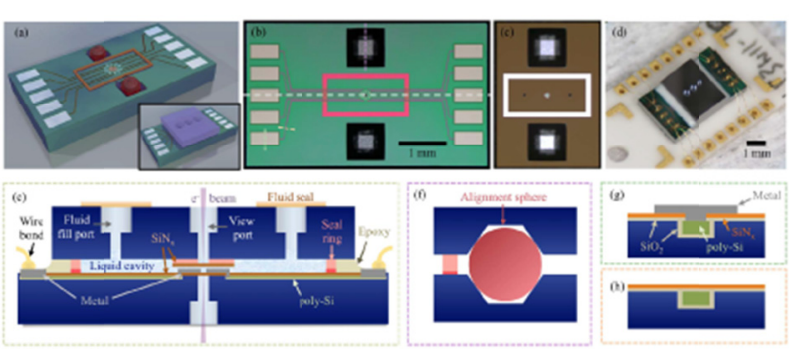

